\title{
Discrimination of POVMs with rank-one effects
}

\author{
Aleksandra Krawiec ${ }^{1}$ (D) $\cdot$ Łukasz Pawela $^{1} \cdot$ Zbigniew Puchała $^{1,2}$
}

Received: 20 February 2020 / Accepted: 23 September 2020 / Published online: 24 November 2020

(c) The Author(s) 2020

\begin{abstract}
The main goal of this work is to provide an insight into the problem of discrimination of positive operator-valued measures with rank-one effects. It is our intention to study multiple-shot discrimination of such measurements, that is the case when we are able to use to unknown measurement a given number of times. Furthermore, we are interested in comparing two possible discrimination schemes: the parallel and adaptive ones. To this end, we construct a pair of symmetric informationally complete positive operatorvalued measures which can be perfectly discriminated in a two-shot adaptive scheme but cannot be distinguished in the parallel scheme. On top of this, we provide an explicit algorithm which allows us to find this adaptive scheme.
\end{abstract}

Keywords Quantum measurements · SIC POVMs · Discrimination of quantum channels

\section{Introduction}

The transformation of quantum states is at the core of quantum computing. The most general mathematical tool describing how quantum states transform are quantum channels. While performing computation, one would like to certify that the operation in use agrees with the one given by the classical description. The discrimination task is closely related to the hypothesis testing. This is one of the reasons why the problem of discrimination of quantum channels has attracted a lot of attention [1-4].

Various approaches have been utilized to study the distinguishability of quantum channels. In work [5], the authors introduced the resource theory of asymmetric distinguishability for quantum channels which was further developed in [6]. A novel

Aleksandra Krawiec

akrawiec@iitis.pl

1 Institute of Theoretical and Applied Informatics, Polish Academy of Sciences, ul. Bałtycka 5, 44-100 Gliwice, Poland

2 Faculty of Physics, Astronomy and Applied Computer Science, Jagiellonian University, ul. Łojasiewicza 11, 30-348 Kraków, Poland 
scenario called coherent quantum channel discrimination was studied in [7]. Interesting results on the distinguishability concern specific classes of quantum channels such as unitary [8,9] and Pauli [10] channels.

We can consider two approaches to channel discrimination - a parallel and an adaptive scheme. In the former, we are allowed to perform the unknown channel a fixed, finite number of times on an input state, while in the latter case, we can also perform additional operations between the applications of the unknown channel. In general, it is a nontrivial question which discrimination scheme should be used to distinguish between two quantum channels. It seems natural that the use of adaptive scheme should improve the discrimination. However, it may happen that it suffices to use the parallel one. For example, in the case of unitary channels [8,9] and von Neumann measurements it was shown in [11-13] that the parallel scheme is always optimal. It is also known that asymptotically the use of adaptive strategy does not give an advantage over the parallel one for the discrimination of classical [14] and classical-quantum channels [15]. Nevertheless, an example of a pair of quantum channels that cannot be distinguished perfectly in parallel but can be distinguished by the adaptive scheme was proposed in [16]. These channels are, however, pretty artificial. We will give another natural example of two channels which have this property. These channels are two symmetric informationally complete positive operator-valued measures (SIC POVMs) $[17,18]$ of dimension three. Moreover, we will show that there exist many pairs of channels which can be distinguished only adaptively and present numerical results for the discrimination of random positive operator-valued measures (POVMs).

In this work, we focus on the following scheme. There are two measurements $\mathcal{P}_{1}$ and $\mathcal{P}_{2}$. One of them is secretly chosen (with probability $\frac{1}{2}$ ) and put into a black box. The black box containing the unknown measurement device can be used any finite number of times in any configuration. Two schemes of multiple-shot discrimination will be studied: parallel and adaptive. The parallel scheme does not require any processing between the uses of the black box [19]. In the adaptive scheme, however, the processing between the uses of the black box plays a crucial role. We can prepare any input state and put it through the discrimination network. Finally, we perform a known measurement and make a decision which measurement was put into the black box.

This work is organized as follows. We begin with preliminaries in Sect. 2. The conditions allowing for perfect discrimination in both parallel and adaptive scenarios are stated in Sect. 3. The example of qutrit SIC POVMs which can be discriminated perfectly only by the adaptive scheme, as well as the algorithm allowing for perfect discrimination, are presented in Sect. 4. Eventually, in Sect 5 one can find numerical results for the discrimination of random rank-one POVMs.

\section{Preliminaries}

Let $\mathcal{X}$ and $\mathcal{Y}$ be finite-dimensional complex Hilbert spaces. We denote by $\mathrm{L}(\mathcal{X}, \mathcal{Y})$ the set of linear operators $A: \mathcal{X} \rightarrow \mathcal{Y}$ and write shortly $\operatorname{L}(\mathcal{X})$ for $\operatorname{L}(\mathcal{X}, \mathcal{X})$. We will use the notation $\mathrm{U}(\mathcal{X}, \mathcal{Y})$ for the set of isometry operators and $\mathrm{U}(\mathcal{X})$ for the set unitary operators. A set of quantum states will be denoted $\mathcal{D}(\mathcal{X})$. Quantum operations 
$\Phi: \mathrm{L}(\mathcal{X}) \rightarrow \mathrm{L}(\mathcal{Y})$ will be denoted $\mathrm{T}(\mathcal{X}, \mathcal{Y})$, while the subset of quantum channels will be denoted $\mathrm{C}(\mathcal{X}, \mathcal{Y})$.

We will need the notion of vectorization of a matrix. For the identity operator $\mathbb{1}$ of the space $\mathrm{L}(\mathcal{X})$, we define its vectorization as $|\mathbb{1}\rangle\rangle=\sum_{i}|i i\rangle$. It can be generalized to an arbitrary matrix $X \in \mathrm{L}(\mathcal{X})$ as $|X\rangle\rangle=(X \otimes \mathbb{1})|\mathbb{1}\rangle\rangle$.

The most general form of quantum measurements are positive operator-valued measures (POVMs). Formally, a collection of positive semidefinite operators $\mathcal{P}=$ $\left\{E_{1}, \ldots, E_{m}\right\} \subset \mathrm{L}(\mathcal{X})$ is called a POVM iff $\sum_{i=1}^{m} E_{i}=\mathbb{1}$. The operators $E_{i}$ are called effects. Every quantum measurement $\mathcal{P}=\left\{E_{1}, \ldots, E_{m}\right\}$ can be identified with a quantum channel which gives a classical output [20]. Its action on a quantum state $\rho$ can be written as

$$
\mathcal{P}(\rho)=\sum_{i=1}^{m} \operatorname{Tr}\left(E_{i} \rho\right)|i\rangle\langle i| .
$$

This approach assumes that the input state ceases to exist, in the sense that it no longer has a defined state and cannot be considered in further calculations. Furthermore, the outcome label $i$ is selected with probability $\operatorname{Tr}\left(E_{i} \rho\right)$.

Let us for a moment focus on the distinguishability of general quantum channels. This problem has been widely studied in the literature in various settings and utilizing a plethora of mathematical tools [21,22]. The probability of correct discrimination between channels $\Phi, \Psi \in \mathrm{C}(\mathcal{X}, \mathcal{Y})$ is upper-bounded by the Holevo-Helstrom theorem [23]

$$
p \leq \frac{1}{2}+\frac{1}{4}\|\Phi-\Psi\|_{\diamond}
$$

The diamond norm, known also as the completely bounded trace norm, $\|\cdot\|_{\diamond}$ is defined for a Hermiticity-preserving linear map $\Phi \in \mathrm{T}(\mathcal{X}, \mathcal{Y})$ as

$$
\|\Phi\|_{\diamond}=\max _{\|X\|_{1}=1}\left\|\left(\Phi \otimes \mathbb{1}_{\mathrm{L}(\mathcal{X})}\right)(X)\right\|_{1}
$$

where $\|X\|_{1}$ is defined as a sum of singular values of $X$. The above-mentioned bound describes the situation when the black box containing the unknown POVM can be used only once. Therefore, $\Phi$ and $\Psi$ are said to be perfectly distinguishable in a single-shot scenario iff $\|\Phi-\Psi\|_{\diamond}=2$.

\subsection{Parallel discrimination}

The approach introduced by Holevo-Helstrom theorem can be easily generalized into the parallel discrimination scheme. In such a case, the probability of correct discrimination after $N$ uses of the black box is upper-bounded by

$$
p \leq \frac{1}{2}+\frac{1}{4}\left\|\Phi^{\otimes N}-\Psi^{\otimes N}\right\|_{\diamond}
$$


Fig. 1 Parallel discrimination scheme

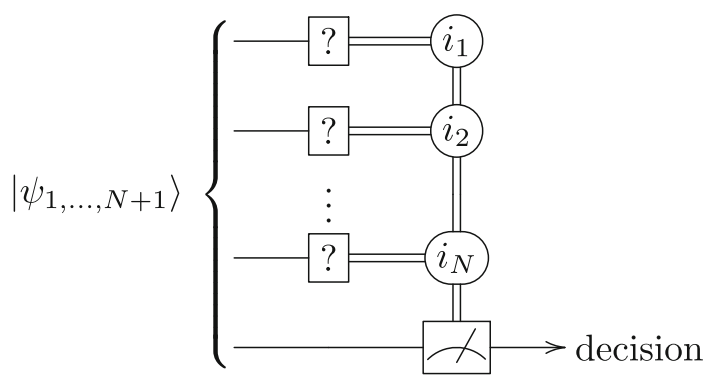

The parallel discrimination scheme is depicted in Fig. 1. Black boxes containing the unknown POVM are denoted by question marks. As an input state, we use the state on $N+1$ registers denoted by $\left|\psi_{1, \ldots, N+1}\right\rangle$. After performing the unknown POVM on $N$ registers, we obtain classical outputs $i_{1}, \ldots, i_{N}$. Using this classical information, we prepare another known measurement and perform it on the last register. Basing on the last measurement's outcome, we make a decision which measurement was hidden in the black box.

We can state a condition when two measurements can be distinguished perfectly in a parallel scenario. It follows directly from Theorem 1 which will be presented in the subsequent section. Let $\mathcal{P}_{1}=\left\{\left|x_{1}\right\rangle\left\langle x_{1}|, \ldots,| x_{m}\right\rangle\left\langle x_{m}\right|\right\}$ and $\mathcal{P}_{2}=$ $\left\{\left|y_{1}\right\rangle\left\langle y_{1}|, \ldots,| y_{m}\right\rangle\left\langle y_{m}\right|\right\}$ be POVMs for $m \geq d$. Then, POVMs $\mathcal{P}_{1}$ and $\mathcal{P}_{2}$ have perfect parallel distinguishability if there exist an integer $N>0$ and a quantum state $\rho \in \mathcal{D}\left(\mathcal{X}^{\otimes N}\right)$ such that for all multi-indices $i_{1}, \ldots, i_{N}$ we have

$$
\left\langle x_{i_{1}} \ldots x_{i_{N}}|\rho| y_{i_{1}} \ldots y_{i_{N}}\right\rangle=0 \text {. }
$$

\subsection{Adaptive discrimination scheme}

If we are allowed to perform processing between the uses of black boxes, then we arrive at the adaptive discrimination scheme, which is depicted in Fig. 2. Although the adaptive discrimination scheme can be used for any number of uses, $N$, of the black box, for the sake of clarity let us focus on the case $N=3$. We prepare an input state $|\psi\rangle$ and perform the unknown POVM on the first register. Basing on the measurement's outcome $i_{1}$, we perform a quantum channel $\Phi_{i_{1}}$ on the other registers. Later, we perform the unknown POVM on the second register and obtain the classical outcome $i_{2}$. Then, we perform a channel $\Phi_{i_{1}, i_{2}}$ on the remaining registers which now depends on both $i_{1}$ and $i_{2}$. In what follows, we perform the unknown POVM on the third register and obtain the outcome $i_{3}$. Finally, we perform the known measurement on the last register which now depends on all classical outcomes $i_{1}, i_{2}, i_{3}$ and make a decision which of the POVMs was hidden in the black box.

\section{Parallel vs adaptive discrimination scheme}

In this section, we will state the condition when a pair of quantum operations can be distinguished perfectly in the adaptive scheme, while the parallel scheme does 


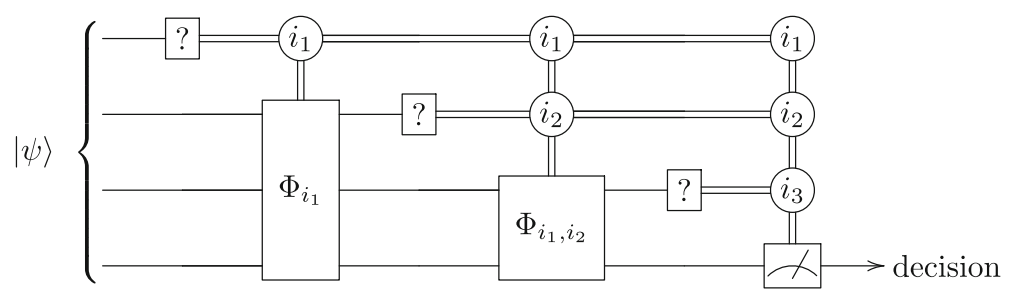

Fig. 2 Adaptive discrimination scheme

not allow for perfect discrimination. Before reviewing some known results on the distinguishability of general quantum operations, we need to introduce the notion of disjointness between quantum operations. Let $\rho \in \mathcal{D}(\mathcal{X})$ be a quantum state with spectral decomposition $\rho=\sum_{i} \lambda_{i}\left|\lambda_{i}\right\rangle\left\langle\lambda_{i}\right|$. Then, we define its support as $\operatorname{supp}(\rho)=$ $\operatorname{span}\left\{\left|\lambda_{i}\right\rangle: \lambda_{i}>0\right\}$.

Definition 1 Two quantum states $\rho_{1}, \rho_{2} \in \mathcal{D}(\mathcal{X})$ are called disjoint when

$$
\operatorname{supp}\left(\rho_{1}\right) \cap \operatorname{supp}\left(\rho_{2}\right)=\{0\}
$$

The notion of disjointness can be generalized to quantum operations. Thus, following [24], we have the following definition.

Definition 2 Two quantum operations $\Phi, \Psi \in \mathrm{T}(\mathcal{X}, \mathcal{Y})$ are called (entanglementassisted) disjoint if there exists an input state $|\psi\rangle \in \mathcal{X} \otimes \mathcal{Z}$ such that $\left(\Phi \otimes \mathbb{1}_{\mathrm{L}(\mathcal{Z})}\right)(|\psi\rangle\langle\psi|)$ and $\left(\Psi \otimes \mathbb{1}_{\mathrm{L}(\mathcal{Z})}\right)(|\psi\rangle\langle\psi|)$ are disjoint.

Let $\Phi, \Psi \in \mathrm{T}(\mathcal{X}, \mathcal{Y})$ be quantum operations with Kraus operators $\left\{E_{i}\right\}_{i}$ and $\left\{F_{i}\right\}_{i}$, respectively. From [24], we have a condition for perfect distinguishability by a finite number of uses given by the following theorem.

Theorem 1 Quantum operations $\Phi: \rho \mapsto \sum_{i} E_{i} \rho E_{i}^{\dagger}$ and $\Psi: \rho \mapsto \sum_{j} F_{j} \rho F_{j}^{\dagger}$ are perfectly distinguishable by a finite number of uses iff they are (entanglement-assisted) disjoint and $\mathbb{1} \notin \operatorname{span}\left\{E_{i}^{\dagger} F_{j}\right\}_{i, j}$.

The following proposition comes from [19] and gives a condition when quantum operations cannot be distinguished in parallel.

Proposition 2 Using notation as in Theorem 1 , if $\operatorname{span}\left\{E_{i}^{\dagger} F_{j}\right\}_{i, j}$ contains a positive operator $\rho>0$, then $\Phi$ and $\Psi$ cannot be distinguished perfectly in parallel by any finite number of uses.

Combining the above Theorem and Proposition, we obtain the corollary which states a condition when operations can be distinguished only by the adaptive scheme, that is, when parallel discrimination is not sufficient but adaptive discrimination allows for perfect discrimination.

Corollary 3 Using notation as in Theorem 1 , if $\operatorname{span}\left\{E_{i}^{\dagger} F_{j}\right\}_{i, j}$ contains a positive operator $\rho>0$, operations $\Phi$ and $\Psi$ are (entanglement-assisted) disjoint and $\mathbb{1} \notin$ $\operatorname{span}\left\{E_{i}^{\dagger} F_{j}\right\}_{i, j}$, then $\Phi$ and $\Psi$ can be distinguished perfectly only by the adaptive scheme. 
Let $\mathcal{P}_{1}$ and $\mathcal{P}_{2}$ be rank-one POVMs with effects $\left\{\left|x_{i}\right\rangle\left\langle x_{i}\right|\right\}_{i}$ and $\left\{\left|y_{i}\right\rangle\left\langle y_{i}\right|\right\}_{i}$, respectively. Therefore, the Kraus operators of $\mathcal{P}_{1}$ and $\mathcal{P}_{2}$ can be chosen as $E_{i}=\left\{|i\rangle\left\langle x_{i}\right|\right\}_{i}$ and $F_{i}=\left\{|i\rangle\left\langle y_{i}\right|\right\}_{i}$, respectively. Hence, in order to check whether $\mathbb{1} \notin \operatorname{span}\left\{E_{i}^{\dagger} F_{j}\right\}_{i, j}$ it suffices to see whether $\mathbb{1} \notin \operatorname{span}\left\{\left|x_{i}\right\rangle\left\langle y_{i}\right|\right\}_{i}$ as $E_{i}^{\dagger} F_{j}=\left|x_{i}\right\rangle\left\langle y_{j}\right| \delta_{i j}$.

\section{SIC POVMs}

In this section, we give an example of a pair of quantum measurements for which the use of parallel discrimination scheme does not give perfect distinguishability but they can be distinguished perfectly by the adaptive scheme. Let us begin with a definition. A POVM $\mathcal{P}=\left\{E_{1}, \ldots, E_{d^{2}}\right\} \subset \mathrm{L}\left(\mathcal{X}_{d}\right)$ of dimension $d$ is called a SIC POVM if $E_{i}=\frac{1}{d}\left|\phi_{i}\right\rangle\left\langle\phi_{i}\right|$ and

$$
\left|\left\langle\phi_{i} \mid \phi_{j}\right\rangle\right|^{2}=\frac{1}{d+1}
$$

for every $i, j=1, \ldots, d^{2}, i \neq j$. It is worth mentioning here that it is an open question whether SIC POVMs exist in all dimensions. So far numerical results are known for all dimensions up to 151 and for a few other dimensions up to 844 [25].

In what follows, we will construct an example of a pair of quantum channels which cannot be distinguished perfectly in the parallel scheme but can be distinguished perfectly in the adaptive scheme. Let $\mathcal{P}_{1}$ be a qutrit SIC POVM with effects $\left\{\left|x_{i}\right\rangle\left\langle x_{i}\right|\right\}_{i}$, where $\left|x_{i}\right\rangle, i=1, \ldots 9$ are columns of the following matrix

$$
\frac{1}{\sqrt{2}}\left[\begin{array}{ccccccccc}
0 & 0 & 0 & -1 & -\omega_{3} & -\omega_{3}^{2} & 1 & 1 & 1 \\
1 & 1 & 1 & 0 & 0 & 0 & -1 & -\omega_{3} & -\omega_{3}^{2} \\
-1 & -\omega_{3} & -\omega_{3}^{2} & 1 & 1 & 1 & 0 & 0 & 0
\end{array}\right]
$$

with $\omega_{3}=\exp \left(\frac{2 \pi \mathrm{i}}{3}\right)$. Let $\pi=(9,8,7,3,1,2,6,4,5)$ be a permutation and $\mathcal{P}_{2}$ be a SIC POVM with effects $\left\{\left|y_{i}\right\rangle\left\langle y_{i}\right|\right\}_{i}$ such that $\left|y_{i}\right\rangle=\left|x_{\pi(i)}\right\rangle$ for $i=1, \ldots, 9$. The construction of the above SIC POVM comes from [26]. In this case, $\mathbb{1} \notin \operatorname{span}\left\{\left|x_{i}\right\rangle\left\langle y_{i}\right|\right\}_{i}$ and $\mathcal{P}_{1}, \mathcal{P}_{2} \in \mathrm{C}(\mathcal{X}, \mathcal{Y})$ are (entanglement-assisted) disjoint. To see the latter, define two states

$$
\begin{aligned}
& \rho_{1}=\left(\mathcal{P}_{1} \otimes \mathbb{1}\right)\left(\frac{1}{d}|\mathbb{1}\rangle\right\rangle\langle\langle\mathbb{1}|)=\frac{1}{d} \sum_{i}|i\rangle\left\langle i|\otimes| x_{i}\right\rangle\left\langle x_{i}\right| \\
& \rho_{2}=\left(\mathcal{P}_{2} \otimes \mathbb{1}\right)\left(\frac{1}{d}|\mathbb{1}\rangle\right\rangle\langle\langle\mathbb{1}|)=\frac{1}{d} \sum_{i}|i\rangle\left\langle i|\otimes| y_{i}\right\rangle\left\langle y_{i}\right| .
\end{aligned}
$$

We need to check whether $\operatorname{supp}\left(\rho_{1}\right) \cap \operatorname{supp}\left(\rho_{2}\right)=\{0\}$. We have

$$
\begin{aligned}
& \operatorname{supp}\left(\rho_{1}\right)=\operatorname{span}\left\{|i\rangle \otimes\left|x_{i}\right\rangle\right\}_{i} \\
& \operatorname{supp}\left(\rho_{2}\right)=\operatorname{span}\left\{|i\rangle \otimes\left|y_{i}\right\rangle\right\}_{i} .
\end{aligned}
$$




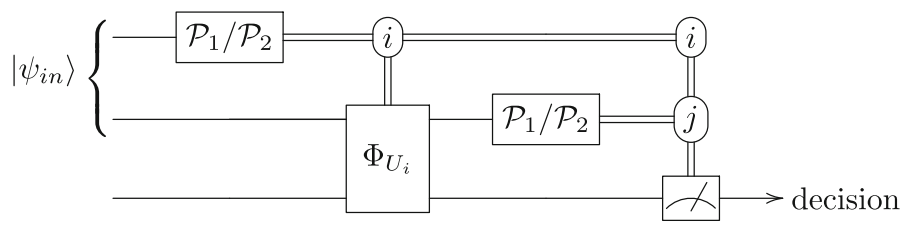

Fig. 3 Adaptive discrimination scheme

Taking $|\varphi\rangle \in \operatorname{supp}\left(\rho_{1}\right) \cap \operatorname{supp}\left(\rho_{2}\right)$, then there exist numbers $\alpha_{1}, \ldots \alpha_{m}$ and $\beta_{1}, \ldots \beta_{m}$ such that

$$
|\varphi\rangle=\sum_{i} \alpha_{i}|i\rangle \otimes\left|x_{i}\right\rangle=\sum_{i} \beta_{i}|i\rangle \otimes\left|y_{i}\right\rangle
$$

As $\left|x_{i}\right\rangle$ and $\left|y_{i}\right\rangle$ are linearly independent for all $i=1, \ldots, k,|\varphi\rangle=0$.

Therefore, from Theorem 1 we obtain that $\mathcal{P}_{1}$ and $\mathcal{P}_{2}$ are perfectly distinguishable by a finite number of uses. Nonexistence of the parallel scheme follows from Proposition 2 , that is, $\mathcal{P}_{1}$ and $\mathcal{P}_{2}$ cannot be distinguished in the parallel scheme as there exists a quantum state $\rho \in \mathcal{D}(\mathcal{X})$ such that $\rho \in \operatorname{span}\left\{\left|x_{i}\right\rangle\left\langle y_{i}\right|\right\}_{i}$. The algorithm finding the state $\rho$ will be presented in Sect. 5 .

Now we focus on the adaptive scheme for perfect discrimination between $\mathcal{P}_{1}$ and $\mathcal{P}_{2}$, which is depicted in Fig. 3. The top register corresponds to the space $\mathcal{X}$, while the second and third registers correspond to spaces $\mathcal{Y}$ and $\mathcal{Z}$, respectively, where each of these spaces is $\mathbb{C}^{3}$. Our goal is as follows. Before the final use of the black box, we would like to obtain states $\left|\xi_{i}\right\rangle$ and $\left|\eta_{i}\right\rangle$ for which $\left(\mathcal{P}_{1} \otimes \mathbb{1}\right)\left(\left|\xi_{i}\right\rangle\left\langle\xi_{i}\right|\right)$ and $\left(\mathcal{P}_{2} \otimes \mathbb{1}\right)\left(\left|\eta_{i}\right\rangle\left\langle\eta_{i}\right|\right)$ are orthogonal. Therefore, we need to find processing $\Phi_{i}$ for which

$$
\begin{aligned}
& \sum_{i}|i\rangle\left\langle i|\otimes| \xi_{i}\right\rangle\left\langle\xi_{i}\left|=\sum_{i}\right| i\right\rangle\langle i| \otimes \Phi_{i}\left(\left(\mathcal{P}_{1} \otimes \mathbb{1}\right)\left(\left|\psi_{i n}\right\rangle\left\langle\psi_{i n}\right|\right)\right), \\
& \sum_{i}|i\rangle\left\langle i|\otimes| \eta_{i}\right\rangle\left\langle\eta_{i}\left|=\sum_{i}\right| i\right\rangle\langle i| \otimes \Phi_{i}\left(\left(\mathcal{P}_{2} \otimes \mathbb{1}\right)\left(\left|\psi_{i n}\right\rangle\left\langle\psi_{i n}\right|\right)\right),
\end{aligned}
$$

where $\left|\psi_{i n}\right\rangle$ is the input state for the discrimination procedure.

The scheme for discrimination between $\mathcal{P}_{1}$ and $\mathcal{P}_{2}$ is as follows. First, using the fact that rank $\left(\operatorname{span}\left\{\left|x_{i}\right\rangle\left\langle y_{i}\right|\right\}_{i}\right)<9$, we can find a nonzero matrix $A \in \mathrm{L}(\mathcal{X})$ such that

$$
A \perp \operatorname{span}\left\{\left|x_{i}\right\rangle\left\langle y_{i}\right|\right\}_{i}
$$

From the singular value decomposition, we can write $A=U \Sigma V^{\dagger}$. Next, find vectors $|\tilde{\xi}\rangle,|\tilde{\eta}\rangle \in \mathcal{Y} \otimes \mathcal{Z}$ as $|\tilde{\xi}\rangle=|U \sqrt{\Sigma}\rangle\rangle$ and $|\tilde{\eta}\rangle=|V \sqrt{\Sigma}\rangle\rangle$. We can see that they fulfill the condition

$$
\operatorname{Tr}_{\mathcal{Z}}|\tilde{\xi}\rangle\langle\tilde{\eta}|=A
$$

Let $|\xi\rangle=\frac{|\tilde{\xi}\rangle}{\| \tilde{\xi}\rangle \|}$ and $|\eta\rangle=\frac{|\tilde{\eta}\rangle}{\||\tilde{\eta}\rangle \|}$. 
Now we present the algorithm for the adaptive discrimination between $\mathcal{P}_{1}$ and $\mathcal{P}_{2}$.

(1) As the input state to the discrimination, we use the maximally entangled state on first two registers, that is, $\left|\psi_{i n}\right\rangle=\frac{1}{\sqrt{d}}|\mathbb{1} \mathcal{X}\rangle$. Then, we perform the unknown measurement on the first register, either $\mathcal{P}_{1}$ or $\mathcal{P}_{2}$, and obtain a label $i$. At the same time on the second register, we obtain the state either $\left|\psi_{i}\right\rangle$ or $\left|\varphi_{i}\right\rangle \in \mathcal{Y}$. Using the property in Eq. (7), one can note that

$$
\left|\left\langle\psi_{i} \mid \varphi_{i}\right\rangle\right|=|\operatorname{Tr}(A)|^{2}=\frac{1}{2}
$$

(2) Basing on the label $i$, we perform an isometric channel $\Phi_{U_{i}}(\cdot)=U_{i} \cdot U_{i}^{\dagger}$ for $U_{i} \in \mathrm{U}(\mathcal{Y}, \mathcal{Y} \otimes \mathcal{Z})$ such that

$$
\begin{aligned}
U_{i}\left|\psi_{i}\right\rangle & =\mathrm{e}^{\mathrm{i} \theta_{i}}|\xi\rangle, \\
U_{i}\left|\varphi_{i}\right\rangle & =|\eta\rangle,
\end{aligned}
$$

where $\theta_{i}=\beta-\alpha_{i}$ for $\left\langle\psi_{i} \mid \varphi_{i}\right\rangle=r_{i} \mathrm{e}^{\mathrm{i} \alpha_{i}}$ and $\langle\xi \mid \eta\rangle=r \mathrm{e}^{\mathrm{i} \beta}$.

(3) After performing the isometric channel, we have a new third register $\mathcal{Z}$. Then, we perform the unknown measurement on the second register $\mathcal{Y}$ and obtain a label $j$.

(4) Finally, we measure the third register by some known measurement and make a decision whether in the black box there was either $\mathcal{P}_{1}$ or $\mathcal{P}_{2}$.

On a final note of this section, we would like to mention that although the above algorithm is described for dimension three, nothing stands in the way to apply it in higher dimensions. We found a permutation of a SIC POVM in dimension four, which can be distinguished adaptively but cannot be distinguished in the parallel scenario. We checked that also in the case of dimension four, our adaptive discrimination procedure can be utilized as well.

\section{Numerical results}

In this section, we study numerically the distinguishability of random POVMs with rank-one effects. We check the conditions for perfect distinguishability. To choose at random a POVM of dimension $d$ with $m$ rank-one effects, we take a Haar random isometry matrix of dimension $d \times m$. Then, we take projectors onto the columns $\left\{\left|x_{i}\right\rangle\right\}_{i=1}^{m}$ of this matrix and obtain a POVM with effects $\left\{\left|x_{i}\right\rangle\left\langle x_{i}\right|\right\}_{i=1}^{m}$.

Let $\mathcal{P}_{1}$ and $\mathcal{P}_{2}$ be POVMs with effects $\left\{\left|x_{i}\right\rangle\left\langle x_{i}\right|\right\}_{i=1}^{m}$ and $\left\{\left|y_{i}\right\rangle\left\langle y_{i}\right|\right\}_{i=1}^{m}$, respectively. If $m=d^{2}$, then $\mathbb{1} \in \operatorname{span}\left\{\left|x_{i}\right\rangle\left\langle y_{i}\right|\right\}$ and therefore any two random rank-one POVMs cannot be distinguished perfectly by any finite number of uses. If $d \leq m<d^{2}$, then $\mathbb{1} \notin$ $\operatorname{span}\left\{\left|x_{i}\right\rangle\left\langle y_{i}\right|\right\}$. Hence, there exists a finite number $N$ of uses which allows for perfect distinguishability iff $\mathcal{P}_{1}$ and $\mathcal{P}_{2}$ are (entanglement-assisted) disjoint. Fortunately, we have the following lemma.

Lemma 4 Consider two independently chosen at random POVMs with rank-one effects. Then, with probability one they are (entanglement-assisted) disjoint. 
Proof Define two states

$$
\begin{aligned}
& \rho_{1}=\left(\mathcal{P}_{1} \otimes \mathbb{1}\right)\left(\frac{1}{d}|\mathbb{1}\rangle\right\rangle\langle\langle\mathbb{1}|)=\frac{1}{d} \sum_{i}|i\rangle\left\langle i|\otimes| x_{i}\right\rangle\left\langle x_{i}\right| \\
& \rho_{2}=\left(\mathcal{P}_{2} \otimes \mathbb{1}\right)\left(\frac{1}{d}|\mathbb{1}\rangle\right\rangle\langle\langle\mathbb{1}|)=\frac{1}{d} \sum_{i}|i\rangle\left\langle i|\otimes| y_{i}\right\rangle\left\langle y_{i}\right| .
\end{aligned}
$$

We need to check whether $\operatorname{supp}\left(\rho_{1}\right) \cap \operatorname{supp}\left(\rho_{2}\right)=\{0\}$. We have

$$
\begin{aligned}
& \operatorname{supp}\left(\rho_{1}\right)=\operatorname{span}\left\{|i\rangle \otimes\left|x_{i}\right\rangle\right\}_{i} \\
& \operatorname{supp}\left(\rho_{2}\right)=\operatorname{span}\left\{|i\rangle \otimes\left|y_{i}\right\rangle\right\}_{i} .
\end{aligned}
$$

Taking $|\varphi\rangle \in \operatorname{supp}\left(\rho_{1}\right) \cap \operatorname{supp}\left(\rho_{2}\right)$, then there exist $\alpha_{1}, \ldots \alpha_{m}$ and $\beta_{1}, \ldots \beta_{m}$ such that

$$
|\varphi\rangle=\sum_{i} \alpha_{i}|i\rangle \otimes\left|x_{i}\right\rangle=\sum_{i} \beta_{i}|i\rangle \otimes\left|y_{i}\right\rangle .
$$

As it holds that for random POVMs $\left|x_{i}\right\rangle$ and $\left|y_{i}\right\rangle$ are linearly independent with probability one for all $i=1, \ldots, k,|\varphi\rangle=0$.

Knowing that for $m<d^{2}$ two POVMs with rank-one effects can always be distinguished perfectly by a finite number of uses, now we are interested when it suffices to use the parallel scheme, instead of the adaptive one. From Proposition 2, we know that if there exists a positive operator $\rho \in \operatorname{span}\left(\left|x_{i}\right\rangle\left\langle y_{i}\right|\right)$, then we need to use the adaptive scheme. Simple numerical calculations show that there exist pairs of qubit POVMs with 3 rank-one effects which can be distinguished perfectly only by the adaptive scheme. Moreover, the probability of finding a pair of POVMs with this property is roughly equal to $\frac{4}{10}$.

Now we proceed to studying the distinguishability of randomly chosen rank-one POVMs in higher dimensions depending on the number of effects. We study this problem numerically for the input dimension $d=7$ and the number of effects $d<$ $m<d^{2}$. The procedure is as follows. We sample $N=10^{6}$ pairs of random POVMs in a manner explained at the beginning of this section. For further details on sampling, we refer the reader to [27,28].

In order to check whether the condition stated in Proposition 2 is fulfilled, we introduce an iterative algorithm. First, we construct a projection operator $P$ on the space $\operatorname{span}\left\{\left|x_{i}\right\rangle\left\langle y_{i}\right|\right\}_{i=1}^{m}$ and choose an initial operator $X \in \mathrm{L}\left(\mathcal{X}_{d}\right)$. We project $X$ onto the subspace given by $P$ obtaining some operator $Y$. We substitute the operator $X$ with the density operator closest to $Y$ and repeat this procedure until it converges or a predefined number of steps is reached. The final step is to check whether the resulting operator is of full rank.

Figure 4 shows numerically found probability of finding a positive operator in the subspace defined in Proposition 2 as a function of the number of effects of POVMs. As we can see in the figure, as the number of effects increases, so does this probability. This is in agreement with previous results. On the one hand, for the case when $m=d$, 
Fig. 4 Numerically estimated lower bound for the probability $p_{\rho}$ of an event that for two randomly sampled POVMs with rank-one effects, the adaptive scenario is necessary for achieving perfect discrimination. The probability is plotted as a function of the number of effects $m$ for the dimension $d=7$. The number of samples per point is $N=10^{6}$

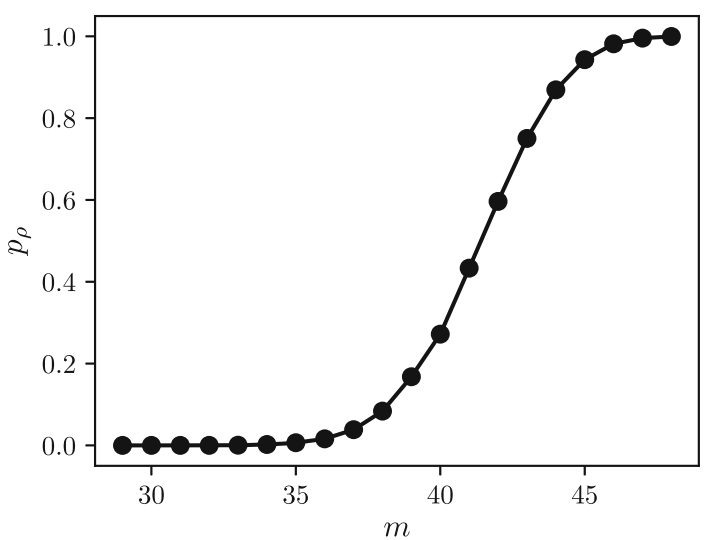

we have a von Neumann POVM and we have shown [13] that such measurements can always be distinguished in a finite number of steps in parallel. On the other hand, when we have $m=d^{2}, \operatorname{span}\left\{\left|x_{i}\right\rangle\left\langle y_{i}\right|\right\}_{i}$ gives the entire space $\mathbb{C}^{d}$ and we are guaranteed to find the identity operator in it, which implies that perfect distinguishability cannot be obtained for any finite number of uses. In general, we are more likely to need to utilize the adaptive scheme for perfect discrimination when the number of effects of POVMs is close to $d^{2}$.

\section{Conclusions}

In this work, we studied the problem of discriminating POVMs with rank-one effects. This class of measurements requires a more careful approach compared to von Neumann POVMs or even general projective measurements. We show that for this class of measurements, there exist instances where the adaptive discrimination scheme is optimal. This is contrary to von Neumann POVMs, for which the parallel scheme is always optimal. We illustrate this result using a special class of POVMs - the symmetric informationally complete POVMs. For a given input dimension $d$, we study the discrimination between a SIC POVM and the same SIC POVM, but with permuted effects. This allows us to construct a simple example of two quantum channels which can be perfectly discriminated in a finite number of steps but require an adaptive scheme. We state the construction of such a discrimination scheme explicitly. Finally, we provide some numerical insight on the probability of randomly sampling a pair of POVMs with rank-one effects which can be distinguished only in the adaptive scenario.

Acknowledgements $\mathrm{AK}$ and $\mathrm{ZP}$ acknowledge financial support by the Foundation for Polish Science through TEAM-NET project (contract no. POIR.04.04.00-00-17C1/18-00). ŁP acknowledges the support of the Polish National Science Centre under the project number 2016/22/E/ST6/00062.

Open Access This article is licensed under a Creative Commons Attribution 4.0 International License, which permits use, sharing, adaptation, distribution and reproduction in any medium or format, as long as you give appropriate credit to the original author(s) and the source, provide a link to the Creative Commons licence, 
and indicate if changes were made. The images or other third party material in this article are included in the article's Creative Commons licence, unless indicated otherwise in a credit line to the material. If material is not included in the article's Creative Commons licence and your intended use is not permitted by statutory regulation or exceeds the permitted use, you will need to obtain permission directly from the copyright holder. To view a copy of this licence, visit http://creativecommons.org/licenses/by/4.0/.

\section{References}

1. D’Ariano, G.M., Presti, P.L., Paris, M.G.: Using entanglement improves the precision of quantum measurements. Phys. Rev. Lett. 87(27), 270404 (2001)

2. Gilchrist, A., Langford, N.K., Nielsen, M.A.: Distance measures to compare real and ideal quantum processes. Phys. Rev. A 71(6), 062310 (2005)

3. Chiribella, G., D’Ariano, G.M., Perinotti, P.: Memory effects in quantum channel discrimination. Phys. Rev. Lett. 101(18), 180501 (2008)

4. Sacchi, M.F.: Optimal discrimination of quantum operations. Phys. Rev. A 71(6), 062340 (2005)

5. Wang, X., Wilde, M.M.: Resource theory of asymmetric distinguishability for quantum channels. Phys. Rev. Res. 1(3), 033169 (2019)

6. Katariya, V., Wilde, M.M.: Evaluating the advantage of adaptive strategies for quantum channel distinguishability. arXiv preprint arXiv:2001.05376 (2020)

7. Wilde, M.M.: Coherent Quantum Channel Discrimination. arXiv preprint arXiv:2001.02668 (2020)

8. Acin, A.: Statistical distinguishability between unitary operations. Phys. Rev. Lett. 87(17), 177901 (2001)

9. Duan, R., Feng, Y., Ying, M.: Entanglement is not necessary for perfect discrimination between unitary operations. Phys. Rev. Lett. 98(10), 100503 (2007)

10. D’Ariano, G.M., Sacchi, M.F., Kahn, J.: Minimax discrimination of two Pauli channels. Phys. Rev. A 72(5), 052302 (2005)

11. Ji, Z., Feng, Y., Duan, R., Ying, M.: Identification and distance measures of measurement apparatus. Phys. Rev. Lett. 96(20), 200401 (2006)

12. Puchała, Z., Pawela, Ł., Krawiec, A., Kukulski, R.: Strategies for optimal single-shot discrimination of quantum measurements. Phys. Rev. A 98(4), 042103 (2018)

13. Puchała, Z., Pawela, Ł., Krawiec, A., Kukulski, R., Oszmaniec, M.: Multiple-shot and unambiguous discrimination of von Neumann measurements. arXiv preprint arXiv:1810.05122 (2018)

14. Hayashi, M.: Discrimination of two channels by adaptive methods and its application to quantum system. IEEE Trans. Inf. Theory 55(8), 3807-3820 (2009)

15. Wilde, M.M., Berta, M., Hirche, C., Kaur, E.: Amortized channel divergence for asymptotic quantum channel discrimination. Lett. Math. Phys. pp. 1-60 (2020)

16. Harrow, A.W., Hassidim, A., Leung, D.W., Watrous, J.: Adaptive versus nonadaptive strategies for quantum channel discrimination. Phys. Rev. A 81(3), 032339 (2010)

17. Renes, J.M., Blume-Kohout, R., Scott, A.J., Caves, C.M.: Symmetric informationally complete quantum measurements. J. Math. Phys. 45(6), 2171-2180 (2004)

18. Flammia, S.T.: On SIC-POVMs in prime dimensions. J. Phys. A: Math. Gen. 39(43), 13483 (2006)

19. Duan, R., Guo, C., Li, C.-K., Li, Y.: Parallel distinguishability of quantum operations. In: 2016 IEEE International Symposium on Information Theory (ISIT), pp. 2259-2263, IEEE, (2016)

20. Watrous, J.: The Theory of Quantum Information. Cambridge University Press, Cambridge (2018)

21. Chiribella, G., D’Ariano, G.M., Perinotti, P.: Quantum circuit architecture. Phys. Rev. Lett. 101(6), 060401 (2008)

22. Chiribella, G., D’Ariano, G.M., Perinotti, P.: Theoretical framework for quantum networks. Phys. Rev. A 80(2), 022339 (2009)

23. Helstrom, C.W.: Quantum Detection and Estimation Theory. Elsevier, Amsterdam (1976)

24. Duan, R., Feng, Y., Ying, M.: Perfect distinguishability of quantum operations. Phys. Rev. Lett. 103(21), 210501 (2009)

25. Fuchs, C.A., Hoang, M.C., Stacey, B.C.: The SIC question: history and state of play. Axioms 6(3), 21 (2017)

26. Dang, H.B., Blanchfield, K., Bengtsson, I., Appleby, D.M.: Linear dependencies in Weyl-Heisenberg orbits. Quantum Inf. Process. 12(11), 3449-3475 (2013) 
27. Gawron, P., Kurzyk, D., Pawela, Ł.: QuantumInformation.jl—a julia package for numerical computation in quantum information theory. PLoS ONE 13, e0209358 (2018)

28. Heinosaari, T., Jivulescu, M.A., Nechita, I.: Random positive operator valued measures. J. Math. Phys. 61(4), $042202(2020)$

Publisher's Note Springer Nature remains neutral with regard to jurisdictional claims in published maps and institutional affiliations. 